\title{
Comparison of Trauma Scoring Systems in Pediatric Trauma Patients
}

\author{
(1) Hasan Sultanoğluำ, (1) Seda Özkan², (1) Tuba Erdem Sultanoğlu³, (1) Nezih Kavak²
}

${ }^{1}$ Clinic of Emergency Medicine, Dr. Ersin Arslan Training and Research Hospital, Gaziantep, Turkey

${ }^{2}$ Clinic of Emergency Medicine, Dışkapı Training and Research Hospital, Ankara, Turkey

${ }^{3}$ Clinic of Physical Therapy and Rehabilitation, Șehitkamil State Hospital, Gaziantep, Turkey

\begin{abstract}
Aim: We aimed to evaluate and compare the performance of BIG score (Base deficit, INR, GCS), pediatric trauma score, revised trauma score, injury severity score, new injury severity score (NISS) in mortality and stay intensive care unit.

Materials and Methods: One thousand five hundred ten pediatric patients aged less than 18 years who were admitted to the emergency department with multi-trauma between 1 July 2012 and 1 July 2016 were included in the retrospective research. Demographic data, vital signs in the emergency department, trauma location, injury severity indexes and follow up of patients were examined.

Results: One thousand five hundred ten patients were included, $40.5 \%$ were female and 59.5\% were male. Mean age was $7.81 \pm 4.8$; mortality was 4.2\%. The best score to evaluate mortality was "probability of survival 2014 (PS14)". The best score to force the stay in ICU was found as NISS, the most sensitive system was NISS and PS14 (94.9\%) and the most specific was NISS (86.7). PS14 was the first to evaluate the survival. In our research, $94.3 \%$ of patient had blunt trauma and $5.7 \%$ had penetrating trauma. PS14 was found the best score to determine survival and mortality for blunt trauma patients.

Conclusion: Although all scoring systems appeared similarly predictive among pediatric trauma patients, The PS14 score was more predictive for mortality and survival, and the NISS score for the need of intensive care admission. The NISS score was the most predictive score for intensive care admission in blunt and penetrating traumas combined. Particularly the newly developed PS14 score can be used as a powerfully predictive scoring system for outcomes among all pediatric trauma patients, irrespective of trauma mechanism.
\end{abstract}

Keywords: Pediatric trauma, trauma scores, BIG, probability of survival 2014, pediatric trauma score, revised trauma score, injury severity score, new injury severity score

\section{Introduction}

Trauma is one of the major causes of death among all age groups. It is the leading cause of death and disability among children older than 1 year of age (1). In addition to designing pre-hospital and hospital trauma organizations, taking meticulous preventive measures and providing public education are greatly important for efforts aimed at reducing trauma-related mortality (2). Initial assessment and management of multi-trauma patients is a difficult task requiring a rapid and systematic approach.
According to the ATLS principles, injured patients are assessed and treated based on their vital signs, level of consciousness, and injury mechanism (3). Additionally, a variety of trauma severity scoring systems has been devised to predict trauma severity and to predict and prevent trauma-related death (4). Trauma severity scoring refers to the process of prediction and quantification of the risks associated with death, hospitalization, and discharge (5). Trauma severity scores assess trauma in terms of its anatomic and/or physiological properties. abbreviated injury scale (AIS) and injury severity score (ISS) take into account injury's anatomic 
properties; Glasgow coma scale (GCS) and revised trauma score (RTS) deals with physiological condition; trauma and injury severity score (TRISS) takes makes a simultaneous assessment of anatomic injury and a patient's physiological condition (6); BIG Score takes into account anatomic and laboratory parameters (7); and PS14 developed by The Trauma Audit and Research Network simultaneously assess anatomic injury and physiological condition (8). Trauma severity scores provide useful guidance for initial pre-hospital assessment, injury severity, patient transportation to an appropriate center, hospital assessment, and mortality prediction.

Here in, we aimed to assess and compare the performances of the BIG Score, PS14, PTS, RTS, ISS, and NISS for predicting mortality and intensive care unit admission in pediatric trauma patients admitted to emergency department. Also, prior to diagnosis of specific injury is important, as they do not therefore help with where patients should go or their resource use in the ED.

\section{Materials and Methods}

This retrospective clinical study was approved by the Local Ethics Committee at Dışkapı Training and Research Hospital (date: 04/04/2016- no: 28/23) and conducted at Dışkapı Training and Research Hospital Emergency Department.

This study included a total of 1510 pediatric patients aged less than 18 years who were admitted to the emergency department with multi-trauma between 1 July 2012 and 1 July 2016 . Patients older than 18 year, with simple trauma and incomplete file records were excluded from the study. Patients' medical information was accessed via hospital automation system and written medical records. Age, sex, nationality, site of trauma, injury type (blunt-penetrating), trauma mechanism, vital signs, and laboratory results were recorded on a previously prepared study form. Patients' Injury severity scores (PTS, AIS, ISS, NISS, GCS, BIG, RTS, PS14), admission to the consulting department, and inhospital outcomes were recorded on patient information forms during their emergency department stay. The newly developed BIG and PS14 scores were calculated as described below:
BIG score: Developed by Borgman et al. (7) in 2011. They retrospectively analyzed data from 2002 to 2009 and showed that base deficit, international normalized ratio (INR), and GCS were correlated to mortality. These variables were formulated as $[$ (base deficit + (INRx2.5) + (15-GCS)] in the BIG scale. This equation was then adapted to a formula predicting mortality. Predicted mortality $=1 /(1+e-x), x=0.2 \times(B I G$ score $)-5.208(9,10)$.

PS14 score: Survival probability of each patient is calculated using logistic regression coefficients. Natural logarithm is used; ISS is converted using the fractional polynomial equation for the model fitting better.

$\mathrm{MCCl}$ represents categorized modified Charlson' Comorbidity index.

$b=i s$ defined as the linear combination of the regression coefficients and related patient's characteristics (ISS, GCS, modified $\mathrm{CCl}$, age and sex) and the constant $\mathrm{e}=2.718282$ (base of Napieran logarithms) (11).

\section{Statistical Analysis}

The statistical analysis of the study data was performed with SPSS-17 software package. Normality of continuous variables was tested with Kolmogorov-Smirnov test. Mann-Whitney U test was used to compare variables that did not meet normality criteria. Surviving and deceased patients were compared using MannWhitney $\mathrm{U}$ test. Penetrating and blunt traumas were separated and compared with Mann-Whitney $U$ test. ROC curves of the trauma scores were drawn using the Med Calc statistical software.

\section{Results}

A total of 1510 patients were included. The mean age of the patients was 7.81 years. Ninety-three percent $(n=1404)$ patients were of Turkish nationality, $6.8 \%(n=102)$ were Syrian, and $0.3 \%$ $(n=4)$ were from other nations. Table 1 summarizes patients' vital signs, laboratory parameters, site of injury, trauma mechanism, and injury location. Nineteen point four percent $(n=293)$ of patients were admitted to the intensive care unit directly from the emergency department or sent to another hospital where

Table 1. Patients' demographic, laboratory parameters, vital signs

\begin{tabular}{lll}
\hline & $\mathbf{n}$ & $\%$ \\
\hline Male & 898 & 59.5 \\
\hline Female & 612 & 40.5 \\
\hline The place where the injury occurred & & \\
\hline Outside & 1069 & 70.8 \\
\hline Nursery and school & 178 & 11.8 \\
\hline Home & 263 & 17.4 \\
\hline Mechanism of trauma & $\mathrm{n}$ & $\%$ \\
\hline In-car traffic accident & 319 & 21.1 \\
\hline Extravehicular traffic accident & 351 & 23.2 \\
\hline Bicycle crash & 133 & 8.8 \\
\hline
\end{tabular}




\section{Table 1. Continued}

\begin{tabular}{|c|c|c|c|}
\hline Motorcycle accident & & 38 & 2.5 \\
\hline Falling from high & & 219 & 14.5 \\
\hline Fall on plain ground & & 101 & 6.7 \\
\hline Falling objects & & 161 & 10.7 \\
\hline Assault & & 93 & 6.2 \\
\hline Cutter tool injury & & 67 & 4.4 \\
\hline Injury with a firearm & & 19 & 1.3 \\
\hline Others & & 9 & 0.6 \\
\hline Injury zone & & $\mathrm{n}$ & $\%$ \\
\hline Head and neck & & 1040 & 68.9 \\
\hline Face & & 557 & 36.9 \\
\hline Thorax & & 636 & 42.1 \\
\hline Abdomen & & 906 & 60 \\
\hline Spine & & 265 & 17.5 \\
\hline Pelvic & & 366 & 24.2 \\
\hline Upper extremity & & 577 & 38.2 \\
\hline Lower extremity & & 579 & 38.3 \\
\hline Vital signs & & Mean \pm SD & Minimum - maximum \\
\hline Age & & $7.8 \pm 4.8$ & $0-18$ \\
\hline Systolic blood pressure (mmHg) & & $89.4 \pm 18.9$ & $0-150$ \\
\hline Diastolic blood pressure (mmHg) & & $63.2 \pm 11.8$ & $0-90$ \\
\hline Pulse (minute) & & $96.9 \pm 21.2$ & $0-161$ \\
\hline Respiratory rate (minute) & & $27.6 \pm 5.6$ & $0-42$ \\
\hline Saturation (\%) & & $93.4 \pm 10.6$ & $0-100$ \\
\hline Laboratory & Mean \pm SD & Minimum - maximum & \\
\hline Leucocyte & $12.4 \pm 5.4$ & $3.6-51.8$ & \\
\hline Hemoglobin & $12.9 \pm 1.6$ & $5-17.7$ & \\
\hline Hematocrit & $39.6 \pm 5.1$ & $15.5-52$ & \\
\hline INR & $1.1 \pm 0.2$ & $0.8-4.5$ & \\
\hline $\mathrm{BE}$ & $-0.7 \pm 4.1$ & $-29.0-11.1$ & \\
\hline Anyon gap & $11.8 \pm 6.9$ & $-26.0-61.5$ & \\
\hline Lactate & $1.8 \pm 1.8$ & $0.1-17$ & \\
\hline Trauma scores & Mean \pm SD & Minimum - maximum & \\
\hline BIG & $7.87 \pm 5.85$ & $2.35-44.8$ & \\
\hline PS14 & $94.18 \pm 16.5$ & $8.84-99.9$ & \\
\hline PTS & $7.51 \pm 3.19$ & $-6-12$ & \\
\hline RTS & $6.77 \pm 1.52$ & $0-7.84$ & \\
\hline ISS & $10.86 \pm 12.25$ & $1-75$ & \\
\hline NISS & $11.38 \pm 13.11$ & $1-75$ & \\
\hline GCS & $12.87 \pm 3.16$ & $3-15$ & \\
\hline Out-come & $\mathrm{n}$ & $\%$ & \\
\hline Discharge from emergency cer & 746 & 49.4 & \\
\hline Admitted to clinic & 312 & 20.7 & \\
\hline Admitted to the intensive care unit & 213 & 14.1 & \\
\hline Referred to another hospital & 177 & 11.7 & \\
\hline Exitus in emergency cervise & 62 & 4.2 & \\
\hline
\end{tabular}

INR: International normalized ratio, PTS: Pediatric trauma score, RTS: Revised trauma score, ISS: Injury severity score, NISS: New injury severity score, GCS: Glasgow coma scale, PS14: Probability of survival 2014, SD: Standard deviation 
they were admitted to intensive care unit. Among patients either admitted to hospital ward or intensive care unit, referred to another center, or treated at the emergency department, 88.6\% $(n=1338)$ were discharged with improvement of their status whereas $11.4 \%(n=172)$ died (Table 1).

The powers of the trauma scores for mortality prediction were analyzed with ROC curves. Accordingly, BIG had an AUC value of $0.984(0.976-0.990)$, a sensitivity of $92.4 \%$, and a specificity of 96.6\%. PS14 score had an AUC of 0.994 (0.988-0.997), a sensitivity of $96.51 \%$, and a specificity of $96.64 \%$. PTS had an AUC of 0.957 (0.946-0.967), a sensitivity of $90.7 \%$, and a specificity of $90.4 \%$. RTS had an AUC of 0.976 (0.967-0.983), a sensitivity of $91.9 \%$, and a specificity of $93.1 \%$. ISS had an AUC of 0.992 (0.9860.996), a sensitivity of $93.6 \%$, and a specificity of $97.3 \%$. NISS had an AUC of 0.993 (0.987-0.997), a sensitivity of $95.9 \%$, and a specificity of $95.5 \%$. GCS had an AUC of 0.987 (0.979-0.992), a sensitivity of $95.4 \%$, and a specificity of $94 \%$ (Figure 1).

Table 2. A comparison of the trauma scores' ROC curves for mortality

\begin{tabular}{lllll}
\hline & AUC & 95\% Accuracy & Sensitivity & Specificity \\
\hline BIG & 0.984 & $0.976-0.990$ & $92.44 \%$ & $96.64 \%$ \\
\hline PS14 & 0.994 & $0.988-0.997$ & $96.51 \%$ & $96.64 \%$ \\
\hline PTS & 0.957 & $0.946-0.967$ & $90.7 \%$ & $90.4 \%$ \\
\hline RTS & 0.976 & $0.967-0.983$ & $91.86 \%$ & $93.12 \%$ \\
\hline ISS & 0.992 & $0.986-0.996$ & $93.6 \%$ & $97.31 \%$ \\
\hline GCS & 0.993 & $0.987-0.997$ & $95.93 \%$ & $95.52 \%$ \\
\hline
\end{tabular}

PS14: Probability of survival 2014, PTS: Pediatric trauma score, RTS: Revised trauma score, ISS: Injury severity score, NISS: New injury severity score

Table 3. A comparison of the trauma scores' ROC curves for intensive care unit

\begin{tabular}{lllll}
\hline & AUC & $95 \%$ Accuracy & Sensitivity & Specificity \\
\hline BIG & 0.895 & $0.878-0.910$ & $83.62 \%$ & $83.98 \%$ \\
\hline PS14 & 0.925 & $0.911-0.938$ & $94.88 \%$ & $82.33 \%$ \\
\hline PTS & 0.851 & $0.832-0.869$ & $75.09 \%$ & $83.24 \%$ \\
\hline RTS & 0.903 & $0.887-0.918$ & $88.05 \%$ & $84.06 \%$ \\
\hline ISS & 0.934 & $0.920-0.946$ & $92.83 \%$ & $88.00 \%$ \\
\hline NISS & 0.936 & $0.923-0.948$ & $94.88 \%$ & $86.69 \%$ \\
\hline GCS & 0.913 & $0.898-0.927$ & $89.76 \%$ & $82.91 \%$ \\
\hline
\end{tabular}

PS14: Probability of survival 2014, PTS: Pediatric trauma score, RTS: Revised trauma score, ISS: Injury severity score, NISS: New injury severity score

Table 4. Comparing trauma scores for blunt and penetrating injuries

\begin{tabular}{llll}
\hline Trauma scores & Blunt & Penetrating & $p$ \\
\hline BIG (mean \pm SD) & $7.98 \pm 5.88$ & $6.13 \pm 5.08$ & 0.000 \\
\hline PS14 (mean \pm SD) & $94.10 \pm 16.53$ & $95.42 \pm 16.13$ & 0.321 \\
\hline PTS (mean, IQR) & 8,4 & 8,2 & 0.356 \\
\hline RTS (mean \pm SD) & $6.76 \pm 1.51$ & $7.09 \pm 1.56$ & 0.003 \\
\hline ISS (mean, IQR) & 6,11 & 3,9 & 0.000 \\
\hline NISS (mean, IQR) & 6,11 & 3,9 & 0.000 \\
\hline GCS (mean, IQR) & 14,3 & 15,1 & 0.000 \\
\hline
\end{tabular}

Mann-Whitney $U$ test, $\mathrm{p}<0.05$ significantly different

SD: Standard deviation, INR: International normalized ratio, PTS: Pediatric trauma score, RTS: Revised trauma score, ISS: Injury severity score, NISS: New injury severity score, GCS: Glasgow coma scale, PS14: Probability of survival 2014 
Table 5. Comparison of ROC curves for mortality prediction in blunt and penetrating trauma

\begin{tabular}{|c|c|c|c|c|}
\hline Blunt & AUC & 95\% Accuracy & Sensitivity & Specificity \\
\hline $\mathrm{BIG}$ & 0.983 & 0.975-0.989 & $92.22 \%$ & $96.42 \%$ \\
\hline PS14 & 0.993 & 0.988-0.997 & $96.41 \%$ & $96.58 \%$ \\
\hline PTS & 0.956 & $0.944-0.966$ & $90.42 \%$ & $89.98 \%$ \\
\hline RTS & 0.975 & 0.966-0.983 & $91.62 \%$ & $93.08 \%$ \\
\hline ISS & 0.992 & 0.986-0.996 & $93.41 \%$ & $97.14 \%$ \\
\hline NISS & 0.993 & 0.987-0.996 & $95.81 \%$ & $95.23 \%$ \\
\hline Penetrating & $A \cup C$ & 95\% Accuracy & Sensitivity & Specificity \\
\hline $\mathrm{BIG}$ & 1.000 & $0.958-1.000$ & $100 \%$ & $100 \%$ \\
\hline PS14 & 1.000 & 0.958-1.000 & $100 \%$ & $100 \%$ \\
\hline PTS & 1.000 & 0.958-1.000 & $100 \%$ & $100 \%$ \\
\hline RTS & 0.995 & 0.949-1.000 & $100 \%$ & $97.53 \%$ \\
\hline ISS & 1.000 & 0.958-1.000 & $100 \%$ & $100 \%$ \\
\hline NISS & 1.000 & $0.958-1.000$ & $100 \%$ & $100 \%$ \\
\hline
\end{tabular}

PTS: Pediatric trauma score, RTS: Revised trauma score, ISS: Injury severity score, NISS: New injury severity score, PS14: Probability of survival 2014

Table 6. A comparison of the scoring systems and the ROC analysis for prediction of intensive care unit admission

\begin{tabular}{|c|c|c|c|c|}
\hline Blunt & AUC & 95\% Accuracy & Sensitivity & Specificity \\
\hline BIG & 0.897 & $0.880-0.912$ & 85.11 & 83.45 \\
\hline PS14 & 0.927 & $0.912-0.940$ & 95.39 & 81.7 \\
\hline PTS & 0.852 & $0.833-0.870$ & 75.89 & 82.92 \\
\hline RTS & 0.905 & $0.888-0.919$ & 87.94 & 83.63 \\
\hline ISS & 0.936 & $0.921-0.948$ & 93.62 & 87.65 \\
\hline NISS & 0.937 & $0.923-0.949$ & 95.04 & 86.34 \\
\hline GCS & 0.914 & $0.899-0.928$ & 91.13 & 82.40 \\
\hline Penetrating & $A \cup C$ & 95\% Accuracy & Sensitivity & Specificity \\
\hline $\mathrm{BIG}$ & 0.882 & $0.795-0.942$ & 100 & 76 \\
\hline PS14 & 0.913 & $0.832-0.963$ & 90.91 & 89.33 \\
\hline PTS & 0.865 & $0.774-0.929$ & 90.91 & 77.33 \\
\hline RTS & 0.865 & $0.774-0.929$ & 90.91 & 90.61 \\
\hline ISS & 0.921 & $0.842-0.968$ & 90.91 & 89.33 \\
\hline NISS & 0.934 & $0.859-0.976$ & 100 & 89.33 \\
\hline GCS & 0.921 & $0.843-0.968$ & 100 & 89.33 \\
\hline
\end{tabular}

PTS: Pediatric trauma score, RTS: Revised trauma score, ISS: Injury severity score, NISS: New injury severity score, PS14: Probability of survival 2014

A comparison of the trauma scores' ROC curves for mortality revealed that the PS14 scoring system had the best sensitivity (Figure 1, Table 2). A comparison of the trauma scores' ROC curves for intensive care unit admission showed that the NISS score had the best sensitivity (Table 3).

Among the study subjects, $5.7 \%(\mathrm{n}=86)$ had penetrating trauma and $94.3 \%(n=1424)$ had blunt trauma. Trauma scores were compared with regard to blunt and penetrating injuries. Although PTS and PS14 scores were not significantly different with respect to blunt and penetrating trauma ( $p>0.05)$, other trauma scores were significantly different $(p<0.05)$ (Table 4). Comparison of ROC curves for mortality prediction in blunt trauma revealed that the NISS and PS14 scoring systems were the most predictive scores. Comparison of ROC curves for mortality prediction in penetrating trauma showed that all scores except for the RTS score were equally predictive (Figure 2, Table 5).

The ROC analysis of GCS for penetrating trauma revealed an AUC value of 1.000 (0.958-1.000), a sensitivity of $100 \%$, and a specificity of $100 \%$ (Figure 2, Table 6). A comparison of the scoring systems for prediction of intensive care unit admission revealed that the NISS and ISS were the most predictive ones (Table 6). 


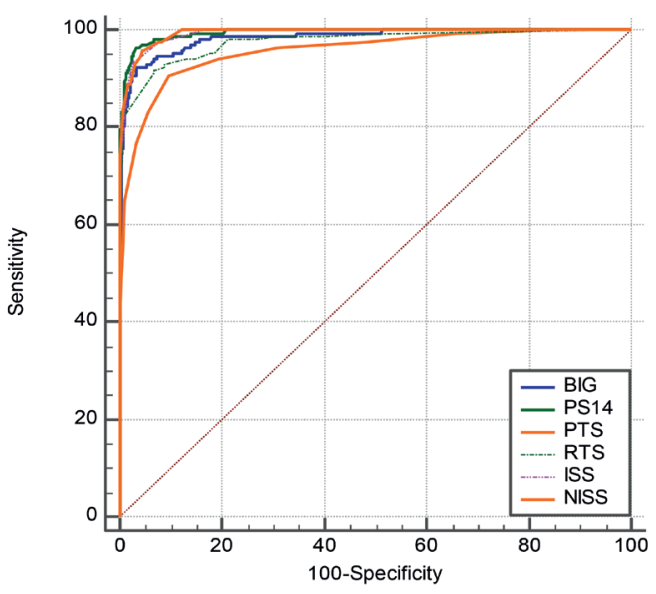

Figure 1. A comparison of the trauma scores' ROC curves for mortality

PTS: Pediatric trauma score, RTS: Revised trauma score, ISS: Injury severity score, NISS: New injury severity score, PS14: Probability of survival 2014
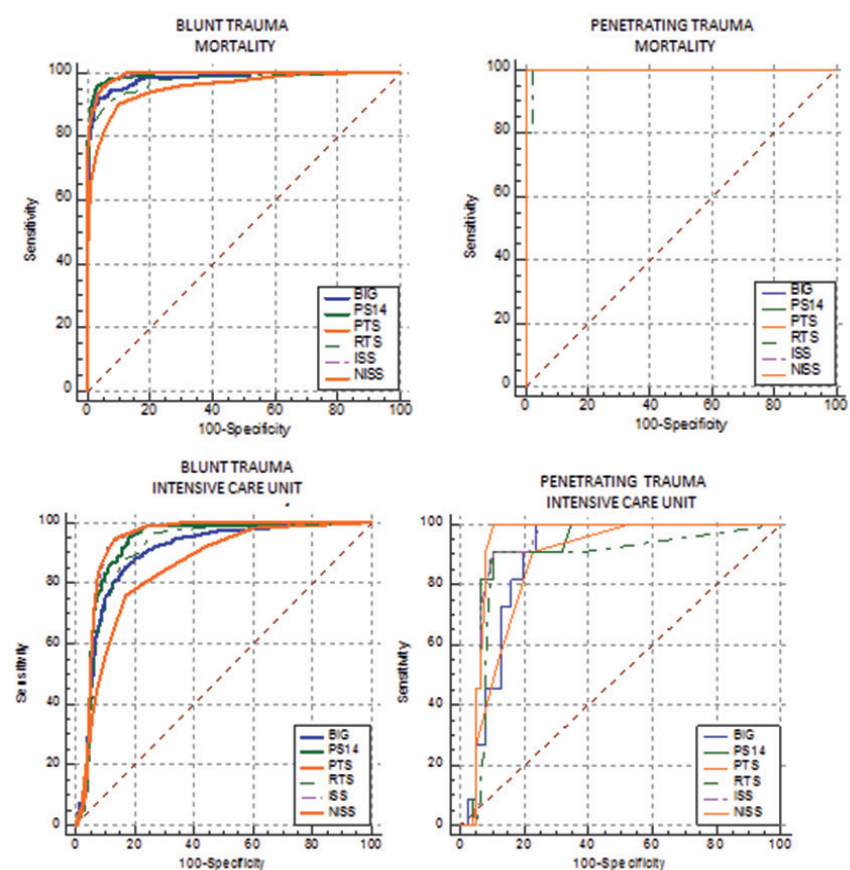

Figure 2. A comparison of the scoring systems and the ROC analysis for prediction of intensive care unit admission

PTS: Pediatric trauma score, RTS: Revised trauma score, ISS: Injury severity score, NISS: New injury severity score, PS14: Probability of survival 2014

\section{Discussion}

Trauma is one of the leading causes of death in all age groups, particularly children. Trauma is the leading cause of death after the age of 4 years in underdeveloped and developing countries and between the ages of 1 and 14 years in developed countries (52\%). Trauma ranks second after infection among as a cause of death between the ages of 1 and 4 years in under-developed regions (9).
BIG score was developed by Borgman et al. (7) in 2011 and first studied in children. Borgman et al. (7) retrospectively analyzed data from 2002 to 2009 and found that base deficit, INR, and GCS were significantly correlated to mortality. Then, they put these variables into the BIG score (base deficit + (INR x2.5) + (15-GCS). In that pediatric study, the BIG score had an AUC of 0.89 for mortality prediction (10). The BIG score was reported to be superior to RTS, ISS, and other pediatric scores used for this indication (7). In 2013 Brockamp et al. (10) used the BIG score in an adult population for the first time. That study compared the BIG, TRISS, PSO9 scores and found that the BIG score was equally predictive for mortality in adults. In line with those studies, we also found an AUC of 0.89 for the BIG score for mortality prediction. The BIG score was more predictive for mortality than the RTS and PTS scores. The most striking advantage of the BIG score compared to other complex scoring systems is its easy calculability with the formula: base deficit + INR + GCS. We are of the opinion that the BIG score can be effectively used for mortality prediction in pediatric trauma patients. In the BIG score developed by Borgman et al. (7) a score of $<12$ predicts a mortality rate of $<5 \%$ and a BIG score of $>26$ is indicative of a mortality risk exceeding $50 \%$. In that study, TRISS and PSO9 showed the best performance when all trauma types were concerned where as the BIG score performed as well as TRISS and PSO9 when penetrating trauma alone was concerned (10). In accordance with the previous studies, our study revealed a mortality rate exceeding 50\% for a BIG score of greater than 26 .

A comparison of ROC curves for mortality pertaining to different trauma scores indicated that PS14 was the most sensitive score (AUC; 0.99, sensitivity; 96.51\%, and specificity; 96.64\%) followed by NISS, ISS, GCS, BIG, RTS, and PTS. A retrospective study involving patients with major bleeding found no significant difference between PS14 and ISS with regard to mortality prediction (11). Assessing a combination of sex, age, ISS, GC, intubation, and comorbidity status as well as including a higher number of mortality-related factors than other scoring systems possibly increased the PS14's predictive power for mortality. Honarmand and Safavi (12) in a study on trauma victims admitted to intensive care unit, showed that NISS predicted the need for intubation and ventilation better than ISS did (12). Lavoie et al. (13) compared ISS and NISS in moderate and severe head trauma and found that NISS was better for predicting the need for intensive care and duration of hospital stay. In our study, the NISS score was the most predictive scoring system for intensive care unit admission (AUC: 0.936). The most sensitive ones were NISS and PS14 (94.8\%), and the most specific one was the NISS (86.69\%). In this sense, our findings were in accordance with previously reported studies. PS14 having the same sensitivity as the NISS system suggests that it can be used as a novel scoring system to predict intensive care unit admission. We are of the opinion that PS14 may be more 
predictive for intensive care unit admission when NISS is used instead of the ISS score, one of the PS14 score's parameters. In a previous study TRISS had an AUC value of 0.88 and ISS 0.67 for predicting survival (14). Our study revealed that PS14 was the most predictive score among others for survival (AUC: 0.99). Solely anatomic or physiological trauma scores remain incapable of predicting trauma-associated outcomes. Thus, PS14 being both an anatomic and physiological index explains its success at predicting trauma outcome.

A comparison of trauma scores for blunt and penetrating injuries showed no difference between PS14 and PTS. Despite being effective for blunt trauma, many trauma scores remain ineffective at assessing penetrating injuries. Our finding suggests that PS14 and PTS can be safely used for penetrating trauma. The reasons of PS14 and PTS having more predictive power in penetrating trauma may include both systems making an anatomic and physiological assessment and the presence of similar parameters in both scores such as PTS taking into account the presence of an open wound, neurological status, and airway while PS14 taking into account ISS, GCS, and intubation status.

A significant correlation was reported between the number of injured organs and mortality and morbidity (15). In blunt trauma PS14 and NISS were the most predictive scores for mortality, with no significant difference having been shown between the two (AUC: 0.99). PS14 had the highest sensitivity (96.41\%) and ISS had the highest specificity (97.14\%) for mortality in blunt trauma. Survival analysis for blunt trauma revealed similar results as mortality, with the PS14 score being the most predictive score. It was considered that PS14 may be as predictive as ISS for mortality in blunt trauma. No study on that subject has been published in the literature.

Many studies published so far have advocated that penetrating trauma is associated with more fatal consequences and more commonly cause organ injuries in children than adults due to the body composition of the former $(16,17)$. In our study RTS alone had an AUC value of 0.995 while the other scores had AUC values of 1.000 for mortality prediction among penetrating trauma patients. In the survival analysis of penetrating traumas, RTS had an AUC value of 0.949 and the others had AUC values of 1.000 .

All scores studied in our study showed similar predictive performances possibly due to the province of our hospital being a pediatric trauma center, cases with higher mortality being referred to our hospital, and our series involving a lower number of patients with penetrating trauma. No previous study has been yet published about this subject.
Borgman et al. (7) reported that TRISS and PSO9 showed the best predictive power when all trauma types are concerned whereas the BIG score showed a similar predictive power for mortality when penetrating trauma alone is concerned.

Ninety percent of children with blunt abdominal trauma does not need any surgical intervention but a close follow-up and intensive care (18). A ROC analysis for the need of intensive care unit admission after blunt trauma showed that the NISS score (AUC: 0.937) was more predictive than the other scores. Moreover, PS14 had the best sensitivity (95.39\%), and ISS had the best specificity (87.65\%). A ROC analysis for the need for intensive care unit admission after penetrating trauma also showed that the NISS score (AUC: 0.934) was predictive. The NISS, BIG, and GCS scores had the best sensitivities (100\%) whereas the RTS had the best specificity. No previous study has been yet published about this subject.

\section{Conclusion}

In conclusion, although all scoring systems appeared similarly predictive among pediatric trauma patients, The PS14 score was more predictive for mortality and survival, and the NISS score for the need of intensive care admission. The NISS score was the most predictive score for intensive care admission in blunt and penetrating traumas combined. Particularly the newly developed PS14 score can be used as a powerfully predictive scoring system for outcomes among all pediatric trauma patients, irrespective of trauma mechanism.

\section{Ethics}

Ethics Committee Approval: This retrospective clinical study was approved by the Local Ethics Committee at Dışkapı Training and Research Hospital (date: 04/04/2016-no: 28/23) and conducted at Dışkapı Training and Research Hospital Emergency Department.

Informed Consent: Retrospective study.

Peer-review: Externally peer-reviewed.

\section{Authorship Contributions}

Concept: H.S., S.Ö., Design: H.S., T.E.S., Data Collection or Processing: H.S., N.K., Analysis or Interpretation: H.S., S.Ö., Literature Search: H.S., S.Ö., Writing: H.S., T.E.S.

Conflict of Interest: No conflict of interest was declared by the authors.

Financial Disclosure: The authors declared that this study received no financial support.

\section{References}

1. National Center for Health Statistics: Health, United States, 2007, with Chartbook on Trendsin the Health of Americans.Hyattsville, MD, U.S. Department of Heath and Human services, Centers for Disease Control and Prevention, National Center for Health Statistics 2007. 
2. Ertekin C, Belgerden S. Travmalı hastaya ilk yaklaşım. Ulus Travma Derg. 1995;1:117-25.

3. American College of Surgeons, Committee on Trauma: Advanced Trauma Life Sup-port for Doctors, Instructor Course Manual, 6th ed. Chicago, American College of Surgeons, 1997.

4. Moore L, Lavoie A, LeSage N, Abdous B, Bergeron E, Liberman M, et al. "Statistical validation of the Revised Trauma Score." J Trauma. 2006;60:305-11.

5. Kilgo PD, Meredith JW, Osler TM. Injury Severity Scoring and Outcomes Research In: Feliciano DV, Mattox KL, Moore EE editors. Trauma, 6th Edition. McGraw-Hill Companies 2008.p.83-90.

6. Erez I, Lazar L, Gutermacher M, Katz S. Abdominal injuries caused by bicycle handlebars. Eur J Surg. 2001;167:331-3.

7. Borgman M, Maegele M, Wade CE, Blackbourne LH, Spinella PC. Pediatric trauma BIG score: predicting mortality in children after military and civilian trauma. Pediatrics. 2011;127:892-7.

8. https://www.tarn.ac.uk/Content.aspx?c=3515.

9. Güloğlu R, Yanar H. Karın Yaralanmaları. Ertekin C, Taviloğlu K, Güloğlu R ve Kurtoğlu M ed. 1. baskı. İstanbul Yayıncılık, 2005.s:875-85.

10. Brockamp T, Maegele M, Gaarder G. Comparison of the predictive performance of the BIG, TRISS, and PS09 score in an adult trauma population derived from multiple international trauma registries. Critical Care. 2013;17:R134.

11. Mothukuri R, Battle C, Guy K, Mills G, Evans PA. The Implementatıon Of Massıve Haemorrhage Protocol (Mhp) For The Management Of Major
Trauma: A Ten Year, Single Centre Study Of Patıent Outcomes. Emergency Medicine Journal. 2015;32:991.

12. Honarmand A, Safavi M. The new Injury Severity Score: a more accurate predictor of need ventilator and time ventilated in trauma patients than the Injury Severity Score. Ulus Travma Acil Cerrahi Derg. 2008;14:110.

13. Lavoie A, Moore L, LeSage N, Liberman M, Sampalis JS. The Injury Severity Score or the New Injury Severity Score for predicting intensive care unit admission and hospital length of stay? Injury. 2005;36:477-83.

14. Rutledge R, Osler T, Emery S, Kromhout-Schiro $S$. The end of the Injury Severity Score (ISS) and the Trauma and Injury Severity Score (TRISS): ICISS, an International Classification of Diseases, ninth revision-based prediction tool, outperforms both ISS and TRISS as predictors of trauma patient survival, hospital charges, and hospital length of stay. J Trauma. 1998;44:41-9.

15. Demircan O, Yağmur Ö, Boğa Z, Erkoçak EU, Alabaz Ö. The Factors Affectıng Postoperatıve Mortalıty In Blunt Abdomınal Trauma Patıents. Ulusal Travma Dergisi. 1995;1:81-5.

16. Cotton BA, Nance ML. Penetrating trauma in children. Semin Pediatr Surg. 2004;13:87.

17. Wessen DE, Stylianos S, Pearl RH. Thoracic injuries, abdominal trauma, in Grosfeld JL, O'neill JA (eds): Pediatric Surgery. 6th Edition, Philadelpia. Mosby Inc. 2006.p:275.

18. Haller JA, Papa P, Drugas G, Colombani P. Nonoperative management of solid organ injuries in children.İ it safe? Ann Surg. 1994;219:625-8. 